\title{
Structured Populations, Linear Semigroups and Positivity
}

\author{
H.J.A.M. Heijmans
}

Centre for Mathematics and Computer Science, Kruislaan 413, NL-1098 SJ Amsterdam, The Netherlands

\section{Introduction}

About twenty-five years ago Garret Birkhoff (1959) indicated how positivity arguments could be exploited in problems from nuclear reactor theory, and he conjectured that infinite dimensional analogues of the Perron-Frobenius theorem would provide the right framework for these kind of problems. Since then it has proved that he was right and nowadays there exists a vast amount of literature where methods from positive operator and semigroup theory are used to study problems in linear transport theory. Moreover, within a few years positive semigroup theory has become a new discipline in functional analysis.

It is the purpose of this paper to indicate how positivity can be exploited succesfully in linear models from structured population dynamics. Diekmann, Heijmans and Thieme (1984) investigate a linear model describing a cell population reproducing by equal fission. The spectrum of the strongly continuous semigroup associated with the problem is obtained (by applying a spectral mapping theorem) from the spectrum of the corresponding generator. This spectrum has been investigated in Heijmans (1985) using the positivity of the resolvent.

In this paper we shall follow a different road, and use the positivity of the semigroup itself. The advantage of this approach is that extensions to nonautonomous situations are possible: e.g. Diekmann, Heijmans \& Thieme (to appear).

This paper consists of the following parts. In Sect. 2 we give a short introduction to positive semigroup theory and prove a very general renewal result. In Sect. 3 we describe a model, covering several examples in structured population dynamics: we shall discuss five of these examples. Then in Sect. 4 we show how a semigroup can be associated with the problem and moreover we shall represent this semigroup as an infinite series. At that place we shall also state our main result concerning the asymptotic behaviour of solutions. In order to prove this main result we apply the theory discussed in Sect. 2 which 
is permitted after some positivity and compactness conditions have been verified. This is successively done in Sect. 5 and Sect. 6. In Sect. 7 we make some final remarks.

\section{Some Results from Positive Semigroup Theory}

Let $X$ be a Banach space and $L: X \rightarrow X$ a closed linear operator. We denote by $\sigma(L), P \sigma(L)$ the spectrum and point spectrum of $L$ respectively. We let $\rho(L)$ be the resolvent set and $r(L)$ the spectral radius. $\mathscr{R}(L)$ and $\mathscr{N}(L)$ denote the range and kernel of $L$ respectively. For a bounded subset $V$ of $X$ the (Kuratowski) measure of noncompactness $\alpha(V)$ is defined as (e.g. Nussbaum (1970)): $\alpha(V)=\inf \left\{d>0 \mid\right.$ there exist a finite number of sets $V_{1}, \ldots, V_{n}$ such that the diameter of $V_{i}$ is less than $d$ and $\left.V=\bigcup_{i=1}^{n} V_{i}\right\}$. The measure of noncompactness $|L|_{\alpha}$ of the bounded linear operator $L: X \rightarrow X$ is by definition

$$
|L|_{\alpha}=\inf \{m \geqq 0 \mid \alpha(L(V)) \leqq m \cdot \alpha(V) \text {, for all bounded subsets } V \text { of } X\} .
$$

The proof of the following result can be found in Nussbaum (1970).

Lemma 2.1. a) $|L|_{\alpha} \leqq\|L\|$ for every bounded linear operator $L$.

b) $\left|L_{1}+L_{2}\right|_{\alpha} \leqq\left|L_{1}\right|_{\alpha}+\left|L_{2}\right|_{\alpha}$ for all bounded linear operators $L_{1}, L_{2}$.

c) $|L+C|_{\alpha}=|L|_{\alpha}$ if $L$ is bounded and $C$ is compact.

Remark 2.2. It follows that $|\cdot|_{\alpha}$ induces a seminorm on the space of bounded linear operators on $X$ (see Nussbaum (1970)).

Definition. The (Browder) essential spectrum $\sigma_{\text {ess }}(L)$ of $L$ is defined by: $\lambda \in \sigma_{\text {ess }}(L)$ if at least one of the following conditions holds

(1) $\lambda$ is a limit point of $\sigma(L)$,

(2) $\mathscr{R}(\lambda I-L)$ is not closed,

(3) $\bigcup_{k \geqq 1} \mathscr{N}\left((\lambda I-L)^{k}\right)$ is infinite dimensional.

Browder (1961) has proved that for a closed operator $L, \lambda \in \sigma(L) \backslash \sigma_{\text {es }}(L)$ implies that $\lambda$ is an eigenvalue of $L$ and, moreover, $\lambda$ is a pole of the resolvent $R(\lambda, L)=(\lambda I-L)^{-1}$ of finite rank. Such an eigenvalue is called a normal eigenvalue. For a bounded operator $L, r_{\text {ess }}(L)$ denotes the radius of the essential spectrum, i.e. $r_{\text {ess }}(L)=\sup \left\{|\lambda| \mid \lambda \in \sigma_{\text {ess }}(L)\right\}$. Nussbaum (1970) proved the following result:

$$
r_{\text {ess }}(L)=\lim _{n \rightarrow \infty}\left|L^{n}\right|_{\alpha}^{\frac{1}{n}} .
$$

Let $A$ be the generator of a strongly continuous semigroup $T(t), t \geqq 0$ (Pazy (1983)). We can properly define

$$
\omega_{0}=\omega_{0}(T(t))=\lim _{t \rightarrow \infty} \frac{1}{t} \log \|T(t)\|,
$$




$$
\omega_{\mathrm{ess}}=\omega_{\mathrm{ess}}(T(t))=\lim _{t \rightarrow \infty} \frac{1}{t} \log |T(t)|_{\alpha}
$$

with the convention that $\log 0=-\infty . \omega_{0}(T(t))$ is called the growth bound and $\omega_{\text {ess }}(T(t))$ the essential growth bound. It can be proved that

$$
r(T(t))=e^{\omega_{0} t}, \quad r_{\mathrm{ess}}(T(t))=e^{\omega_{\mathrm{ess}} t}, \quad t>0,
$$

where $e^{-\infty}=0$. The spectral bound $s(A)$ of the generator $A$ is defined by

$$
s(A)=\{\operatorname{Re} \lambda \mid \lambda \in \sigma(A)\},
$$

where $s(A)=-\infty$ if this set is empty. Then

$$
\omega_{0}(T(t))=\max \left\{s(A), \omega_{\text {ess }}(T(t))\right\} .
$$

These results can be found in Prüss (1981) in a slightly different formulation (see also Webb (1985)). A standard result in semigroup theory (e.g. Webb (1985)) says:

For all $\omega>\omega_{0}$ there is $M(\omega) \geqq 1$ such that $\|T(t)\| \leqq M(\omega) e^{\omega t}$, for all $t \geqq 0$.

A question which is very important in many applications is whether or not $s(A)=\omega_{0}(T(t))$ and how the peripheral spectrum $\sigma_{+}(A)$ of $A,\left(\sigma_{+}(A)\right.$ $=\{\lambda \in \sigma(A) \mid \operatorname{Re} \lambda=s(A)\}$ if $s(A)>-\infty$ and $\sigma_{+}(A)=\varnothing$ if $\left.s(A)=-\infty\right)$ looks like. Very precise answers to these questions are known for so-called positive semigroups. Let us first give some definitions. For the rest of this section we assume that $X$ is a Banach lattice and we let $X_{+}$be the cone of positive elements (Schaefer (1974)). We denote by $X^{*}$ the dual space and by $X_{+}^{*}$ the dual cone. Finally we let $\langle F, \phi\rangle$ be the duality pairing for $\phi \in X, F \in X^{*}$.

Definition. The semigroup $T(t)$ is called positive (i.e. $T(t) \geqq 0)$ if $T(t)$ leaves the cone invariant for all $t \geqq 0$. We call $T(t)$ irreducible if for every $\phi \in X_{+}, \phi \neq 0$, $F \in X_{+}^{*}, F \neq 0$ there exists a $t>0$ such that $\langle F, T(t) \phi\rangle>0$.

Remark 2.3. Proposition III.8.3 of Schaefer (1974) shows that this definition is equivalent to Schaefer's original definition.

The following theorem has been proved by Greiner (1981).

Theorem 2.4. Let $A$ be the generator of a positive irreducible semigroup $T(t)$ and suppose that $s(A)$ is a pole of the resolvent, then $\sigma_{+}(A)=s(A)+i \alpha \mathbb{Z}$ for some real $\alpha \geqq 0$, and every element $s(A)+i \alpha k, k \in \mathbb{Z}$ is a pole of order one of the resolvent, and moreover its geometric multiplicity is one.

We can now characterize the peripheral spectrum $\sigma_{+}(A)$ under an additional assumption. Compare Corollary 1.7 of Greiner (1984a).

Theorem 2.5. Let $T(t)$ be a positive irreducible semigroup with generator $A$ such that $s(A)$ is a pole of the resolvent. Suppose moreover that

$$
\omega_{0}(T(t))>\omega_{\text {ess }}(T(t))
$$

then there is an $\varepsilon>0$ such that $\operatorname{Re} \lambda<s(A)-\varepsilon$ for all $\lambda \in \sigma(A), \lambda \neq s(A)$. 
Proof. Since the part of the spectrum at the right of the vertical line $\operatorname{Re} \lambda$ $=\omega_{\text {ess }}(T(t))$ contains only (normal) eigenvalues and therefore is "faithfull" to the spectrum of the semigroup (Pazy (1983)), i.e. $\left\{e^{t \lambda} \mid \lambda \in \sigma(A), \quad \operatorname{Re} \lambda>\omega_{\text {ess }}\right\}$ $=\left\{\mu \in \sigma(T(t))|| \mu \mid>r_{\text {ess }}(T(t))\right\}$ it follows that it suffices to show that $\sigma_{+}(A)$ $=\{s(A)\}$. Suppose not. Then $\sigma_{+}(A)=s(A)+i \alpha \mathbb{Z}$ for some $\alpha \geqq 0$. This implies that the closure of the set $\left\{e^{s(A) t} e^{i \alpha k t} \mid k \in \mathbb{Z}\right\}$ is contained in $\sigma(T(t))$. If $\alpha t / \pi$ is irrational (and this is true for a.e. $t>0$ ) then $\left\{\mu|| \mu \mid=e^{s(A) t}\right\} \subseteq \sigma(T(t)$ ), yielding that $\omega_{\text {ess }}(T(t)) \geqq s(A)$ which contradicts $\omega_{\text {ess }}(T(t))<\omega_{0}(T(t))$.

Let $\gamma=s(A)$ and let $\psi_{0}, F_{0}$ be the associated positive eigenvector and adjoint eigenvector,

$$
A \psi_{0}=\gamma \psi_{0}, \quad A^{*} F_{0}=\gamma F_{0},
$$

normalized by the conditions

$$
\left\|\psi_{0}\right\|=1, \quad\left\langle F_{0}, \psi_{0}\right\rangle=1 .
$$

Remark 2.6. If for example $X=L^{1}(\mu)$ for some $\sigma$-finite measure space $(\Omega, \Sigma, \mu)$, then $\psi_{0}$ and $F_{0}$ are positive a.e. (Schaefer (1974)).

We let $P_{0}$ be the one-dimensional strictly positive projection

$$
P_{0} \phi=\left(F_{0} \otimes \psi_{0}\right) \phi \stackrel{\text { def }}{=}\left\langle F_{0}, \phi\right\rangle \psi_{0}, \quad \phi \in X .
$$

The large time behaviour of solutions $n(t)=T(t) \phi$ of the abstract Cauchy problem

$$
\frac{d n}{d t}=A n, \quad n(0)=\phi
$$

is characterized by the following theorem.

Theorem 2.7. Let $A$ be the generator of the positive, irreducible semigroup $T(t)$ for which the inequality $\omega_{\mathrm{ess}}(T(t))$ holds. Then there exist constants $\varepsilon, M>0$ such that for all $\phi \in X$ the following estimate holds.

$$
\left\|e^{-\gamma t} T(t) \phi-P_{0} \phi\right\| \leqq M e^{-\varepsilon t}\|\phi\|, \quad t \geqq 0
$$

where $\gamma$ and $P_{0}$ are defined above.

Proof. Let $\varepsilon>0$ be determined by Theorem 2.5 such that $\gamma-\varepsilon>\omega_{\text {ess }}(T(t))$. If $\mu \in \sigma(T(t)), \mu \neq e^{\gamma t}$ then $|\mu|<e^{(\gamma-\varepsilon) t}$. Let $Z=\mathscr{R}\left(e^{\gamma t} I-T(t)\right)$ (which does not depend on $t$ ) and let $T_{Z}(t)$ denote the restriction of $T(t)$ to $Z$, then $T_{Z}(t)$ defines a strongly continuous semigroup. Since $\sigma\left(T_{Z}(t)\right)=\sigma(T(t)) \backslash\left\{e^{\gamma t}\right\}$, we have $r\left(T_{Z}(t)\right)<e^{(\gamma-\varepsilon) t}, \quad t>0$. Therefore $\omega_{0}\left(T_{Z}(t)\right)<\gamma-\varepsilon$ and we get that $\left\|T_{Z}(t) \phi\right\| \leqq M e^{(\gamma-\varepsilon) t}\|\phi\|, \phi \in Z$ for some positive constant $M$ (see (2.6)). Now let $\phi \in X$. Clearly $\phi=P_{0} \phi+\left(I-P_{0}\right) \phi, T(t) P_{0} \phi=e^{\gamma t} P_{0} \phi$ and $\left\|T(t)\left(I-P_{0}\right) \phi\right\|=\| T_{Z}(t)$ $\left(I-P_{0}\right) \phi\left\|\leqq M e^{(\gamma-\varepsilon) t}\right\|\left(I-P_{0}\right) \phi\left\|\leqq M e^{(\gamma-\varepsilon) t}\right\| \phi \|$. This yields the result.

In the subsequent sections we shall apply these results to a concrete problem in structured population dynamics. 


\section{The Model and Some Examples}

Consider a biological population whose individuals are completely characterized by the one- dimensional quantity $x$. We say that $x$ is the state of the individual and we assume that $[0,1]$ is the state space of the population: this means among others that indeed all states $0 \leqq x \leqq 1$ (with the possible exception of 0 and 1) can be reached eventually by some individual. We assume that individuals with state $x \geqq \Delta$ can jump instanteneously to some lower state $x-\Delta$ where $0<\Delta<1$ is a fixed parameter.

Let $n(t, x)$ be the function representing the distribution of the individuals over all individual states $x \in[0,1]$ at time $t$, i.e. $\int_{x_{1}}^{x_{2}} n(t, x) d x$ is the number of individuals with state between $x_{1}$ and $x_{2}$ at time $t$. Let $\phi(x)$ denote the state distribution at time $t=0$, then $n(t, x)$ can be computed from:

$$
\frac{\partial n}{\partial t}(t, x)+\frac{\partial}{\partial x}(g(x) n(t, x))=\sigma(x) n(t, x)-b(x) n(t, x)+b(x+\Delta) n(t, x+\Delta),
$$

where one should read $b(x+\Delta) n(t, x+\Delta)=0$ if $x+\Delta>1$,

$$
\begin{aligned}
g(0) n(t, 0) & =\int_{0}^{1} h(x) n(t, x) d x, \\
n(0, x) & =\phi(x) .
\end{aligned}
$$

Here $g(x)$ denotes the growth rate accounting for the fact that between two jumps the state of an individual increases continuously according to the ordinary differential equation

$$
\frac{d x}{d t}=g(x)
$$

$\sigma(x)$ denotes the entrance (if $\sigma(x) \geqq 0$ ) - disappearance (if $\sigma(x)<0$ ) rate; a very well known example of disappearance is provided by death. $b(x)$ is the jump rate and $h(x)$ the reproduction rate. The boundary condition (3.1b) expresses the fact that all newborns obtain the state $x=0$ at birth. We refer to the lecture notes edited by Metz and Diekmann (in prep.) where it is explained in great detail how to derive balance equations constituting structured population models.

We define $X(t, x)$ as the state of an individual at time $t$ given that its state at time zero was $x$ and no jumps have occurred meanwhile. Then $X(t, x)$ is the solution of the ordinary differential equation

$$
\frac{d X}{d t}=g(X), \quad X(0, x)=x .
$$

The curves $t \mapsto(t, X(t, x))$, where $0 \leqq x<1$, are the characteristic curves of (3.1 a).

We shall study the initial value problem (3.1) in $L^{1}[0,1]$, which seems to be the most natural choice. So we assume that $\phi \in L^{1}[0,1]$. Let $n(t)$ be given by $n(t)(x)=n(t, x), x \in[0,1]$. We call $n(t, x)$ a solution of (3.1) if and only if 
(1) $n(t) \in L^{1}[0,1], t \geqq 0$ and $t \mapsto n(t)$ is continuous as a mapping from $\mathbb{R}_{+}$ into $L^{1}[0,1]$.

(2) $n$ is differentiable along the characteristics of (3.1 a), i.e. for all $t>0$ and $0<x<1$

$$
(D n)(t, x)=\lim _{h \rightarrow 0}\left[\frac{g(X(h, x)) n(t+h, X(h, x))-g(x) n(t, x)}{h}\right] \quad \text { exists. }
$$

(3) For all $t>0$ and $0<x<1$

$$
\begin{aligned}
\frac{1}{g(x)} \cdot(D n)(t, x) & =\sigma(x) n(t, x)-b(x) n(t, x)+b(x+\Delta) n(t, x+\Delta), \\
n(t, 0) & =\int_{0}^{1} h(x) n(t, x) d x, \quad t>0, \\
n(0, x) & =\phi(x), \quad 0 \leqq x \leqq 1 .
\end{aligned}
$$

Remark 3.1. In the probability-theoretic literature (3.1a)-(3.1b) is called the forward equation. In some problems it seems biologically more relevant and/or mathematically easier to study the associated backward equation (for instance in the space of continuous functions). For an example we refer to Heijmans (1984b) where we study the "backward formulation" of the problem described in Example 3.9.

In order to obtain a well-defined mathematical problem we have to make some assumptions, which fortunately do hardly limitate the applicability to biological models.

Assumption 3.2. $\sigma, h, b$ and $g$ are continuously differentiable on $[0,1]$. Moreover $h, b$ and $g$ are nonnegative.

Smoothness is assumed to keep the forthcoming analysis surveyable. Without doubt it can be weakened without yielding essentially new biological phenomena (see Sect. 7). Positivity of $h, b$ and $g$ follows immediately from the biological interpretation.

Assumption 3.3 a) $g(x)>0, x \in[0,1), g(1)=0$ and $g^{\prime}(1) \neq 0$.

b) $g(x+\Delta)<g(x), 0 \leqq x \leqq 1-\Delta$.

This is our most important and also most restrictive assumption. A biological implication is that an individual can never reach state $x=1$; a generation (see Sect. 4 for a precise definition) never becomes extinct, and this has some very important mathematical consequences. Observe that assumption $3.3 \mathrm{a} \mathrm{im-}$ plies that assumption $3.3 \mathrm{~b}$ is satisfied in a neighbourhood of $x=1-\Delta$. Biologically, assumption $3.3 \mathrm{~b}$ means the following. Consider two individuals, both with state $x>\Delta$ at time $t=0$. The first individual jumps immediately to state $x$ $-\Delta$ and finally reaches state $x_{1}=X(t, x-\Delta)$ at time $t$. The second individual starts growing first, and subsequently jumps back to state $x_{2}=X(t, x)-\Delta$ at time $t$. If $g(x+\Delta) \neq g(x)$ for all $x$ then $x_{1} \neq x_{2}$. This implies that the jump process provides a dispersion mechanism, separating individuals of one cohort. 
Before stating our third assumption we have to give some definitions. Let $a_{h} \in[0,1]$ be the smallest value such that: $h(x)=0, x \in\left(a_{h}, 1\right]$. Let $a_{b} \in[\Delta, 1]$ be given by $a_{b}=\min \left\{a_{h}+\Delta, 1\right\}$.

Assumption 3.4. If $a_{h}<1$ then $b(x)>0$ on $\left[a_{b}, 1\right] \cap(\Delta, 1]$.

If $b(1)=0$ then $\int_{0}^{1} \frac{h(x)}{g(x)} \exp \left(-\int_{0}^{x} \frac{b(\xi)-\sigma(\xi)-v}{g(\xi)} d \xi\right) d x>1$,
where $v=b(1)-\sigma(1)$.

The first part of assumption 3.4 says that every individual without regard to its state has the possibility to reproduce at some future time instant (perhaps after one or more jumps) or to reach a state arbitrarily close to zero. In other words: every state between 0 and 1 is reachable for an individual or its progeny. This implies that we have to do our bookkeeping on the whole individual state space $[0,1]$ if we are interested in the time dependent development of the population. The second part of assumption 3.4 is rather technical; it is needed to settle estimate (2.7). It is easily seen that this last assumption is fulfilled if $b(1)+h(1)>0$.

We shall now describe five examples from structured population dynamics which can be reduced to system (3.1) by choosing a suitable new state description. Only in the first example we shall indicate how the assumptions 3.23.4 are carried over to the new situation. In the other examples this is left to the reader.

Example 3.5. Size Dependent Cell Growth. Consider a population of unicellular organisms whose members are characterized by their size $s$. The population reproduces by fission into two equal parts and the rate at which cells with size $s$ divide is given by $\beta(s)$. We assume that $\beta$ is $C^{1}, \beta(s)=0$ if $s \leqq a$ (where $0<a<1)$ and $\beta(s)>0$ if $s>a$. Then the minimum possible size is $\frac{1}{2} a$. Let individual cell growth be governed by

$$
\frac{d s}{d t}=\gamma(s)
$$

where $\gamma$ is a $C^{1}$-function, $\gamma(s)>0$ if $\frac{1}{2} a \leqq s<1, \gamma(1)=0$ and $\gamma^{\prime}(1) \neq 0$.

Finally we assume that the mortality rate $\mu=\mu(s)$ is a non-negative $C^{1}$ function. Let $N_{0}(s), N(t, s)$ be the size distribution at time zero and time $t$ respectively:

$$
\begin{aligned}
\frac{\partial N}{\partial t}(t, s)+\frac{\partial}{\partial s}(\gamma(s) N(t, s))=- & \mu(s) N(t, s)-\beta(s) N(t, s)+4 \beta(2 s) N(t, 2 s) \\
& N\left(t, \frac{1}{2} a\right)=0 \\
& N(0, s)=N_{0}(s)
\end{aligned}
$$

We define the state variable $x$ by

$$
x=\chi(s)=1-\frac{\log s}{\log \frac{1}{2} a} .
$$


The inverse function $s=S(x)$ is given by

$$
s=S(x)=2^{-\left(\frac{1-x}{4}\right)},
$$

where $\Delta=-\frac{\log 2}{\log \frac{1}{2} a}>0$. If we put

$$
n(t, x)=S^{\prime}(x) \cdot S(x) \cdot N(t, S(x)),
$$

then (3.3) transforms into

$$
\begin{aligned}
\frac{\partial n}{\partial t}(t, x)+\frac{\partial}{\partial x}(g(x) n(t, x))=\sigma(x) & n(t, x)-b(x) n(t, x)+b(x+\Delta) n(t, x+\Delta), \\
n(t, 0) & =0 \\
n(0, x) & =\phi(x),
\end{aligned}
$$

where $g(x)=\frac{\gamma(S(x))}{S^{\prime}(x)}, \sigma(x)=-\mu(S(x))+\frac{\gamma(S(x))}{S(x)}$ and $b(x)=\beta(S(x))$, and where we have used that $S^{\prime}(x+\Delta)=2 S^{\prime}(x)$. Therefore this model fits into our framework.

Remark 3.6. a) Observe that $\int_{1_{1}}^{x_{2}} n(t, x) d x$ is not a number, but a biomass since it follows from (3.4) that $\int_{x_{1}}^{x_{2}} n(t, x) d x=\int_{s_{1}}^{s_{2}} s N(t, s) d s$, where $s_{i}=S\left(x_{i}\right), i=1,2$.

b) The cell division problem (3.3) has been extensively investigated by Diekmann et al. (1984) for the case that growth remains bounded away from zero in a neighbourhood of 1 , but instead the rate $\beta(s)$ becomes infinite in such a way that $\int_{a}^{1} \beta(s) d s=\infty$. In that case the cell cycle time is finite for all cells, whereas this is not true in the model under consideration.

Example 3.7. Reproduction Causing a Decrease in Weight. Consider a population whose individuals are characterized by their weight $w$ which varies between $w_{0}$ and $w_{1}$. An adult having weight $w \geqq(r+1) w_{0}$ (where $r$ is fixed and $\left.(r+1) w_{0}<w_{1}\right)$ can give birth to $r$ offspring (for instance eggs) all having the same weight $w_{0}$, thereby reducing its own weight to $w-r \cdot w_{0}$. Let $\beta=\beta(w)$ be the reproduction rate which is identically zero on $\left[w_{0},(r+1) w_{0}\right]$, and let $\mu, \gamma, N_{0}$ and $N$ have the same interpretation as in the previous example. The following equations hold:

$$
\begin{aligned}
\frac{\partial N}{\partial t}(t, w)+\frac{\partial}{\partial w}(\gamma(w) N(t, w))= & -\mu(w) N(t, w)-\beta(w) N(t, w) \\
& +\beta\left(w+r w_{0}\right) N\left(t, w+r w_{0}\right), \\
\gamma\left(w_{0}\right) N\left(t, w_{0}\right)= & r \int_{(r+1) w_{0}}^{w_{1}} \beta(w) N(t, w) d w, \\
N(0, w)= & N_{0}(w) .
\end{aligned}
$$


If we define the new state $x$ as: $x=\frac{w-w_{0}}{w_{1}-w_{0}}$, then this model provides a second example if $\beta, \mu$ and $\gamma$ obey the conditions associated with assumptions 3.2-3.4.

Example 3.8. Populations Subject to Random Catastrophes. Consider a collection of populations. We assume that an individual (in our terminology this is a population which is a member of the collection) is characterized by the quantity $s$, denoting the size of the population. Furthermore we assume that every individual is subject to growth,

$$
\frac{d s}{d t}=\gamma(s)
$$

and to random catastrophes, which reduce the population size from $s$ to $p \cdot s$ where $p \in(0,1)$ is fixed. We denote by $\beta(s)$ the rate at which catastrophes occur and we assume that there exists a number $a \in(0,1)$ such that $\beta(s)>0, s>a$ and $\beta(s)=0$ elsewhere. With respect to $\gamma$ we make the meanwhile well-known assumptions:

$$
\gamma \in C^{1}, \quad \gamma(s)>0 \quad \text { if } s<1, \quad \gamma(1)=0, \quad \gamma^{\prime}(1) \neq 0 .
$$

Let $N(t, s)$ be the size distribution then $N$ obeys

$$
\begin{aligned}
\frac{\partial N}{\partial t}(t, s)+\frac{\partial}{\partial s}(\gamma(s) N(t, s)) & =-\beta(s) N(t, s)+\frac{1}{p} \beta\left(\frac{s}{p}\right) N\left(t, \frac{s}{p}\right), \\
N(t, p a) & =0, \\
N(0, s) & =N_{0}(s),
\end{aligned}
$$

where $N_{0}$ is the initial size distribution. A similar transformation as in Example 3.5 carries the problem over into (3.1). We refer to Gripenberg (1983) for a different approach.

Example 3.9. Holling's Hungry Mantid Model. A fourth example is given by the equation describing the probability distribution $N(t, s)$ of the satiations $s$ of an invertebrate predator catching preys with fixed weight $w$ at a rate $\beta(s)$.

$$
\begin{gathered}
\frac{\partial N}{\partial t}(t, s)-\frac{\partial}{\partial s}(c s N(t, s))=-\beta(s) N(t, s)+\beta(s-w) N(t, s-w), \\
N\left(t, s_{\max }\right)=0 \\
N(0, s)=N_{0}(s),
\end{gathered}
$$

where the satiation $s, 0 \leqq s \leqq s_{\max }$ between two catches decreases exponentially with time

$$
\frac{d s}{d t}=-c \cdot s
$$


We refer to Metz and van Batenburg (1985) and Heijmans (1984b) for more details.

In this case one can define the new state $x$ by: $x=1-\frac{s}{S_{\max }}$.

Example 3.10. Age Structured Populations. Our final example is studied extensively in the literature (see Prüss (1981), Webb (1985). It concerns the growth of an age-structured population on an infinite age-interval which excludes the situation that the reproduction rate $\beta(a)$, where $a$ is age, obeys $\beta(a)=0, a \geqq A$. To reduce this problem to our formulation one can define the new state $x$ by: $x=1-e^{-\theta a}$, where $\theta>0$ is fixed. Then

$$
g(x)=\frac{d x}{d t}=\frac{d x}{d a}=\theta e^{-\theta a}=\theta(1-x) .
$$

\section{Semigroup Solution to the Problem and the Main Result}

We can rewrite (3.1) as an abstract Cauchy problem on the space $L^{1}[0,1]$ :

$$
\frac{d n}{d t}=A n, \quad n(0)=\phi,
$$

where the closed operator $A$ is given by

$$
(A \psi)(x)=-\frac{d}{d x}(g(x) \psi(x))+\sigma(x) \psi(x)-b(x) \psi(x)+b(x+\Delta) \psi(x+\Delta),
$$

for all $\psi$ in the domain $\mathscr{D}(A)$ of $A$,

$$
\begin{gathered}
\mathscr{D}(A)=\left\{\psi \in L^{1}[0,1] \mid g \cdot \psi\right. \text { is absolutely continuous and } \\
\left.g(0) \psi(0)=\int_{0}^{1} h(x) \psi(x) d x\right\},
\end{gathered}
$$

which is densely defined. In this section we shall prove that $A$ generates a strongly continuous semigroup $T(t)$ and we shall give a series representation of this semigroup. First we write $A$ as the sum of a closed operator $B$,

$$
(B \psi)(x)=-\frac{d}{d x}(g(x) \psi(x))+\sigma(x) \psi(x)-b(x) \psi(x),
$$

having the same domain as $A$, and a bounded operator $C$ given by

$$
(C \psi)(x)= \begin{cases}b(x+\Delta) \psi(x+\Delta), & 0 \leqq x \leqq 1-\Delta, \\ 0, & x>1-\Delta .\end{cases}
$$

A straightforward computation shows that $B$ is the generator of a strongly continuous semigroup, and now a standard result from semigroup theory (Pazy 
(1983)) says that $A$ being the sum of $B$ and a bounded operator $C$, also generates a strongly continuous semigroup which we denote by $T(t)$. The solution $n(t)=T(t) \phi$ of (4.1) can be represented by the series

$$
n(t)=\sum_{i=0}^{\infty} n_{i}(t) \stackrel{\text { def }}{=} \sum_{i=0}^{\infty} T_{i}(t) \phi,
$$

where the $n_{i}$ 's are obtained from

if $i=0$, and

$$
\begin{aligned}
& \frac{\partial n_{0}}{\partial t}+\frac{\partial\left(g n_{0}\right)}{\partial x}=(\sigma(x)-b(x)) n_{0}(t, x) \\
& g(0) n_{0}(t, 0)=0 \\
& n_{0}(0, x)=\phi(x)
\end{aligned}
$$

$$
\begin{aligned}
& \frac{\partial n_{i}}{\partial t}+\frac{\partial\left(g n_{i}\right)}{\partial x}=(\sigma(x)-b(x)) n_{i}(t, x)+b(x+\Delta) n_{i-1}(t, x+\Delta) \\
& g(0) n_{i}(t, 0)=\int_{0}^{1} h(x) n_{i-1}(t, x) d x \\
& n_{i}(0, x)=0
\end{aligned}
$$

if $i \geqq 1$.

Remark 4.1. Let $S_{0}(t)$ be the semigroup generated by $B$, then a variation-ofconstants formula applied to $\frac{d n}{d t}=B n+C n$, with $C n$ being considered as the inhomogeneous part of the equation, reduces the Cauchy problem (4.1) to the integral equation $n(t)=S_{0}(t) \phi+\int_{0}^{t} S_{0}(t-\tau) C n(\tau) d \tau$, from which by the method of successive approximations, $n(t)$ is found to be $n(t)=\sum_{i=0}^{\infty} S_{i}(t) \phi$, where $S_{i}(t)$ can be obtained from $S_{0}(t)$ and $S_{i-1}(t)$ by means of the formula $S_{i}(t) \phi=$ $\int_{0}^{t} S_{0}(t-\tau) C S_{i-1}(\tau) \phi d \tau, i \geqq 1$. The above expansion is different from the one given in (4.6) in the sense that the computation of $T_{0}(t) \phi$ involves the boundary condition $g(0) n(t, 0)=0$ whereas the computation of $S_{0}(t) \phi$ involves $g(0) n(t, 0)=\int_{0}^{1} h(x) n(t, x) d x$ and something similar holds for the other terms $T_{i}(t), S_{i}(t)$.

Now we shall reformulate the initial value problem (3.1) as an integral equation from which all terms $n_{i}(t)$ in (4.6) can be computed. We pretend as if the expressions $b(x+\Delta) n(t, x+\Delta)$ and $\int_{0}^{1} h(x) n(t, x) d x$ in (3.1) are known à priori, and compute the solution of the thus obtained inhomogeneous equation. As a result we find the following integral equation:

$$
\begin{aligned}
n(t, x)= & \frac{E(x)}{g(x)}\left\{\frac{g(X(-t, x))}{E(X(-t, x))} \phi(X(-t, x))+\int_{0}^{1} h(\xi) n(t-\tau(x), \xi) d \xi\right. \\
& \left.+\int_{0}^{t} \frac{g(X(-\tau, x))}{E(X(-\tau, x))} b(X(-\tau, x)+\Delta) n(t-\tau, X(-\tau, x)+\Delta) d \tau\right\},
\end{aligned}
$$


where

$$
\begin{aligned}
\tau(x) & =\int_{0}^{x} \frac{d \xi}{g(\xi)} \\
E(x) & =\exp \left(\int_{0}^{x} \frac{\sigma(\xi)-b(\xi)}{g(\xi)} d \xi\right),
\end{aligned}
$$

(i.e. $\tau(x)$ is the time an individual needs to grow from state 0 to $x$ ) where one should read $n(t, x)=0$ if $t<0$, and take a function of $x$ to be zero if its argument is not in $[0,1]$. Now $n_{i}(t, x)$ can be computed from the formulae

$$
\begin{aligned}
n_{0}(t, x)= & \frac{E(x)}{g(x)} \cdot \frac{g(X(-t, x))}{E(X(-t, x))} \cdot \phi(X(-t, x)), \\
n_{i}(t, x)= & \frac{E(x)}{g(x)}\left\{\int_{0}^{1} h(\xi) n_{i-1}(t-\tau(x), \xi) d \xi+\int_{0}^{t} \frac{g(X(-\tau, x))}{E(X(-\tau, x))}\right. \\
& \left.\cdot b(X(-\tau, x)+\Delta) n_{i-1}(t-\tau, X(-\tau, x)+\Delta) d \tau\right\},
\end{aligned}
$$

with the same conventions as in (4.9). Observe that $T_{0}(t)$ defines a strongly continuous semigroup.

Each function $n_{i}$ has a clear biological interpretation. $n_{0}$ represents the members of the 0 'th generation, i.e. those individuals present at time zero which have not experienced a jump yet. The $i$ 'th generation, represented by $n_{i}$, consists of the offspring of members of the $(i-1)$ 'th generation, and those individuals who were members of the $(i-1)$ 'th generation at an earlier time, but have experienced one jump during the time elapsed. Observe from (4.12) that a generation, once it has come into existence never goes extinct anymore.

The two following sections are concerned with the verification of the conditions of Theorem 2.7:

i) $T(t)$ is a positive, irreducible semigroup.

ii) $\omega_{\mathrm{ess}}(T(t))<\omega_{0}(T(t))$.

We can state our main result now.

Theorem 4.2. There exists a constant $\gamma \in \mathbb{R}$, a strictly positive projection $P_{0}$ of rank 1 and positive constants $M, \varepsilon>0$ such that for all $\phi \in L^{1}[0,1]$

$$
\left\|e^{-\gamma t} T(t) \phi-P_{0} \phi\right\| \leqq M e^{-\varepsilon t}\|\phi\|, \quad t>0
$$

Moreover $P_{0}$ can be represented as $P_{0}=F_{0} \otimes \psi_{0}$, where $F_{0} \in L^{\infty}[0,1]$ and $\psi_{0} \in L^{1}[0,1]$ are positive a.e.

This (renewal) theorem says that the population grows or decays exponentially (depending on the sign of $\gamma$ ) and the $x$-distribution becomes stationary if $t \rightarrow \infty$. At $t=\infty$ the dependence on the initial data is only reflected by the constant $\left\langle F_{0}, \phi\right\rangle$. 


\section{Irreducibility of the Semigroup}

In this section we shall establish irreducibility of $T(t)$ with respect to the cone $L_{+}^{1}[0,1]$, consisting of all nonnegative functions of $L^{1}[0,1]$. Actually we shall prove a much stronger result here. Let $X(t, x)$ be as in Sect. 3 .

Theorem 5.1. There is a $t^{*}>0$ such that $n(t, x)>0,0<x<X\left(t-t^{*}, 0\right), t>t^{*}$.

The rest of this section is devoted to the proof of this result. Let us assume for simplicity that the initial function $\phi$ is continuous and $\phi(x)>0, x \in\left(\xi_{0}^{-}, \xi_{0}^{+}\right)$, where $0<\xi_{0}^{-}<\xi_{0}^{+}<1$. This assumption does not mean a restriction of generality since one can easily see from (4.12) that $n_{1}(t, x)$ indeed obeys this assumption if $t$ is large enough. Let $\xi^{-}(t)=X\left(t, \xi_{0}^{-}\right)$and $\xi^{+}(t)=X\left(t, \xi_{0}^{+}\right)$and we define $T(x, y)$ as the time which an individual needs to grow from $x$ to $y$, i.e. $T(x, y)$ $=\tau(y)-\tau(x)$ where $\tau$ is given by (4.10).

Lemma 5.2. $n_{0}(t, x)>0, \xi^{-}(t)<x<\xi^{+}(t), t>0$.

This result follows immediately from (4.12a).

Lemma 5.3. If $n(t, x)>0, x_{1}<x<x_{2}$, where $a_{b}<x_{1}<x_{2}<1$, then $n(t, x)>0$, $x_{1}-\Delta<x<x_{2}-\Delta$.

This result can easily be verified, using integral equation (4.9).

Lemma 5.4. If $a_{h}<1$ then there exists a $t_{0}>0$ such that $n(t, 1-\Delta)>0$ if $t>t_{0}$.

Proof. It follows from assumption 3.4 that $b(x)>0$ on $(1-\varepsilon, 1]$ for some $\varepsilon>0$. We choose $t_{0}^{\prime} \geqq 0$ such that $\xi^{-}\left(t_{0}^{\prime}\right)>1-\varepsilon$. Let $t_{0}=t_{0}^{\prime}+T\left(\xi^{-}\left(t_{0}^{\prime}\right)-\Delta, 1-\Delta\right)$. Since $X(0,1-\Delta)+\Delta=1>\xi^{+}(t)$ and $X\left(-t+t_{0}^{\prime}, 1-\Delta\right)+\Delta<X\left(-t_{0}+t_{0}^{\prime}, 1-\Delta\right)+\Delta$ $=\xi^{-}\left(t_{0}^{\prime}\right)$, and from the continuity of $X(., 1-\Delta), \xi^{-}($.$) and \xi^{+}($.$) it follows that$ there exist $\tau_{1}(t), \tau_{2}(t)$ for $t>t_{0}$ such that: 1

i) $0<\tau_{1}(t)<\tau_{2}(t) \leqq t-t_{0}^{\prime}$

ii) if $\tau \in\left(\tau_{1}(t), \tau_{2}(t)\right)$ then $X(-\tau, 1-\Delta)+\Delta \in\left(\xi^{-}(t-\tau), \xi^{+}(t-\tau)\right)$.

Thus $n(t, 1-\Delta) \geqq n_{1}(t, 1-\Delta) \geqq \int_{\tau_{1}(t)}^{\tau_{2}(t)}$ (something positive). $\quad\left(\frac{g}{E} \phi\right)(X(-t+\tau$, $X(-\tau, 1-\Delta)+\Delta)) d \tau>0, t>t_{0}$, since $X(-t+\tau, X(-\tau, 1-\Delta)+\Delta) \in\left(\xi_{0}^{-}, \xi_{0}^{+}\right)$if $\tau \in\left(\tau_{1}(t), \tau_{2}(t)\right)$. This yields the result.

Proof of Theorem 5.1. We have to distinguish between three cases:

i) $a_{h}=0$. Let $t_{0}$ be such that $n(t, 1-\Delta)>0, t>t_{0}$. Let $q \in \mathbb{N}$ and $\delta>0$ be such that $1-\Delta \leqq q \Delta<1$ and $q \Delta+\delta<1$. From the integral equation (4.9) it follows that $n(t, x)>0$ if $x \in[1-\Delta, q \Delta+\delta)$ and $t>t_{0}+T(1-\Delta, q \Delta+\delta)=t^{*}$. Now we obtain from Lemma 5.3 that $n(t, x)>0$ if $x \in(0, \delta)$ and $t>t^{*}$. Thus $n(t, x)>0$ if $x \in\left(0, X\left(t-t^{*}, 0\right)\right), t>t^{*}$.

ii) $0<a_{h}<1$. Using Lemmata 5.4 and 5.3 one can easily show that there is a $\delta, 0<\delta<a_{h}$ and $t^{*}>0$ such that $n(t, x)>0, x \in\left(a_{h}-\delta, a_{h}\right), t>t^{*}$. From the definition of $a_{h}$ and the integral equation (4.9) we obtain that $n(t, 0)>0$ if $t>t^{*}$ and therefore $n(t, x)>0, x \in\left[0, X\left(t-t^{*}, 0\right)\right), t>t^{*}$. 
iii) $a_{h}=1$. The definition of $a_{h}$ yields that for $t^{*}$ large, $h$ is not identically zero on $\left(\xi^{-}(t), \xi^{+}(t)\right)$ if $t>t^{*}$, and from Lemma 5.2 we may conclude that $n(t, 0)>0$ if $t>t^{*}$. Consequently $n(t, x)>0$ if $x \in\left(0, X\left(t-t^{*}, 0\right)\right), t>t^{*}$, and the result is proved.

Corollary 5.5. $T(t)$ is irreducible.

Remark 5.6. It is striking that the proof of this result does not require assumption $3.3 \mathrm{~b}$. However to some extent this is an optical illusion since this assumption has some relation with the condition $g(1)=0$, which is heavily exploited in the proof. Corollary 5.5 can also be proved by using Proposition D.3 of Voigt (1984).

\section{The Inequality $\omega_{\text {ess }}(T(t))<\omega_{0}(T(t))$}

One cannot expect the inequality

$$
\omega_{\text {ess }}(T(t))<\omega_{0}(T(t))
$$

to be true if not some sort of compactness of $T(t)$ can be established. We write $T(t)=T_{0}(t)+U(t)$, where $U(t)=\sum_{i=1}^{\infty} T_{i}(t)$ and $T_{i}(t), i \geqq 0$ is defined in Sect. 4 .

Lemma 6.1. $U(t)$ is compact for all $t \geqq 0$.

Proof. It suffices to show that $T_{1}(t)$ is continuous with respect to the uniform operator topology and compact, since all the other terms $T_{i}(t)$ are obtained from $T_{1}(t)$ by integration. From (4.12) we obtain that

$$
\begin{aligned}
\left(T_{1}(t) \phi\right)(x)= & n_{1}(t, x)=\frac{E(x)}{g(x)} \cdot\left\{\int_{0}^{1} h(y) \frac{E(y)}{g(y)} \cdot\left(\frac{g}{E} \cdot \phi\right)(X(-t+\tau(x), y)) d y\right. \\
+ & \int_{0}^{t} \frac{g(X(-\tau, x))}{E(X(-\tau, x))} \cdot\left(b \cdot \frac{E}{g}\right)(X(-\tau, x)+\Delta) \\
& \left.\cdot\left(\frac{g}{E} \cdot \phi\right)(X(-t+\tau, X(-\tau, x)+\Delta)) d \tau\right\},
\end{aligned}
$$

with the same conventions as in formula (4.9). We use an Arzéla-Ascoli-like theorem to prove compactness. The first term is easy. In the second term we substitute $\xi=X(-t+\tau, X(-\tau, x)+\Delta)$ and a simple calculation shows that

$$
\frac{d \tau}{d \xi}=\frac{g(X(-\tau, x)+\Delta)}{g(\xi) \cdot\{g(X(-\tau, x)+\Delta)-g(X(-\tau, x))\}}
$$

and this expression never becomes zero if assumption $3.3 \mathrm{~b}$ is satisfied. Now, after making some tedious but straightforward estimates, compactness and continuity with respect to the uniform operator topology follow.

We obtain from Lemma 2.1 that

$$
|T(t)|_{\alpha}=\left|T_{0}(t)+U(t)\right|_{\alpha}=\left|T_{0}(t)\right|_{\alpha} \leqq\left\|T_{0}(t)\right\|,
$$


and from (2.2), (2.3) we get

$$
\omega_{\text {ess }}(T(t)) \leqq \omega_{0}\left(T_{0}(t)\right) .
$$

$\omega_{0}\left(T_{0}(t)\right)$ is obtained from the following lemma. Let

$$
v=b(1)-\sigma(1) \text {. }
$$

Lemma 6.2. There exist constants $m, M>0$ such that

$$
m e^{-v t} \leqq\left\|T_{0}(t)\right\| \leqq M e^{-v t}, \quad t \geqq 0 .
$$

Proof. It follows from assumptions 3.2 and $3.3 \mathrm{a}$ that there exist positive constants $m_{1}, m_{2}, m_{3}, m_{4}$ such that $m_{1}(1-x) e^{-c t} \leqq 1-X(t, x) \leqq m_{2}(1-x) e^{-c t}$ and $m_{3}(1-x)^{\frac{v}{c}} \leqq E(x) \leqq m_{4}(1-x)^{\frac{v}{c}}$, where $c=-g^{\prime}(1)>0$. From (4.12a) we obtain that

$$
\begin{aligned}
\left\|T_{0}(t) \phi\right\| & =\int_{0}^{1}\left|n_{0}(t, x)\right| d x=\int_{X(0, t)}^{1} \frac{E(x)}{g(x)} \cdot\left(\frac{g}{E} \cdot|\phi|\right)(X(-t, x)) d x \\
& =\int_{0}^{1} \frac{E(X(t, \xi))}{E(\xi)}|\phi(\xi)| d \xi \leqq \int_{0}^{1} \frac{m_{4}\left(m_{2}(1-\xi) e^{-c t}\right)^{\frac{v}{c}}}{m_{3}(1-\xi)^{\frac{v}{c}}}|\phi(\xi)| d \xi=M e^{-v t}\|\phi\|,
\end{aligned}
$$

where $M=\frac{m_{4}}{m_{3}} \cdot m_{2}^{\frac{v}{c}}$. Similarly $\left\|T_{0}(t) \phi\right\| \geqq m e^{-v t}\|\phi\|$, where $m=\frac{m_{3}}{m_{4}} \cdot m_{1}^{\frac{v}{c}}$.

This result implies that $\omega_{0}\left(T_{0}(t)\right)=-v$ and from (6.2) we get

$$
\omega_{\mathrm{ess}}(T(t)) \leqq-v,
$$

(it is not difficult to show that the equality holds) and therefore we "only" have to prove that

or equivalently (see (2.5))

$$
\omega_{0}(T(t))>-v
$$

$$
s(A)>-v,
$$

in order to settle (6.1). The rest of this section is concerned with the proof of (6.6). The reader should observe that this is the only place where the generator plays an essential role.

Let $f \in L^{1}[0,1]$. The inhomogeneous equation $\lambda \psi-A \psi=f$ can be reduced to

$$
\psi(x)=\frac{E_{\lambda}(x)}{g(x)} \cdot\left\{\int_{0}^{1} h(\xi) \psi(\xi) d \xi+\int_{0}^{x} \frac{b(\xi+\Delta)}{E_{\lambda}(\xi)} \psi(\xi+\Delta) d \xi+\int_{0}^{x} \frac{f(\xi)}{E_{\lambda}(\xi)} d \xi\right\}
$$

where

$$
E_{\lambda}(x)=E(x) e^{-\lambda \tau(x)}
$$


As in the proof of the former lemma we can show that there exist positive constants $l_{1}, l_{2}$ such that

$$
l_{1}(1-x)^{\frac{v+\operatorname{Re} \lambda}{c}} \leqq\left|E_{\lambda}(x)\right| \leqq l_{2}(1-x)^{\frac{v+\operatorname{Re} \lambda}{c}},
$$

and these estimates yield that the separate terms at the right-hand-side of (6.7) only make sense (i.e. define $L^{1}$-functions) if $v+\operatorname{Re} \lambda>0$.

Now, for all $\lambda \in \mathbb{C}$ with $v+\operatorname{Re} \lambda>0$ we define the bounded operators $T_{\lambda}, U_{\lambda}$ on $L^{1}[0,1]$ by

$$
\begin{gathered}
\left(T_{\lambda} \phi\right)(x)=\frac{E_{\lambda}(x)}{g(x)} \cdot\left\{\int_{0}^{1} h(\xi) \phi(\xi) d \xi+\int_{0}^{x} \frac{b(\xi+\Delta)}{E_{\lambda}(\xi)} \phi(\xi+\Delta) d \xi\right\}, \\
\left(U_{\lambda} \phi\right)(x)=\frac{E_{\lambda}(x)}{g(x)} \int_{0}^{x} \frac{\phi(\xi)}{E_{\lambda}(\xi)} d \xi, \quad \phi \in L^{1}[0,1] .
\end{gathered}
$$

The following result is straightforward.

Lemma 6.3. If $\nu+\operatorname{Re} \lambda>0$ then $T_{\lambda}$ and $U_{\lambda}$ are compact.

Now for $\lambda \in \rho(A)$, i.e. the resolvent set of $A$, we have $(\lambda I-A)^{-1} f=$ $\left(I-T_{\lambda}\right)^{-1} U_{\lambda} f$ and we conclude that for all $\lambda$ with $\nu+\operatorname{Re} \lambda>0$ we have

$$
\lambda \in \sigma(A) \Leftrightarrow \lambda \in P \sigma(A) \Leftrightarrow 1 \in \sigma\left(T_{\lambda}\right) .
$$

Now suppose that $\lambda$ is real and $v+\lambda>0$ then $T_{\lambda}$ is positive and a famous result of Krein and Rutman (1948) says that the spectral radius $r_{\lambda}=r\left(T_{\lambda}\right)$ is an eigenvalue. Thus, if there exists a $\lambda_{0}>-v$ such that $r\left(T_{\lambda_{0}}\right)=1$, then $\lambda_{0} \in \sigma(A)$, and therefore $s(A) \geqq \lambda_{0}>-v$ and in that case we are done. Since $\lambda \rightarrow r\left(T_{\lambda}\right)$ is continuous and

$$
\lim _{\lambda \rightarrow \infty} r\left(T_{\lambda}\right)=0,
$$

as one can show quite easily, it suffices to prove that

$$
\lim _{\lambda \downarrow-\nu} r\left(T_{\lambda}\right)>1 \text {. }
$$

To this end we shall use the following result due to Krein and Rutman (1948).

Lemma 6.4. If $L$ is a positive operator and $\psi$ a positive, nonzero vector such that $L \psi \geqq c \cdot \psi$, for some positive constant $c$, then $r(L) \geqq c$.

We shall distinguish between two cases.

(i) $b(1)>0$. Let $\lambda \in \mathbb{R}, v+\lambda>0$. Some simple estimations using (6.9) show that there is a positive constant $C$ such that

$$
\left(T_{\lambda} \psi\right)(x) \geqq C(1-x)^{\nu+\lambda-1} \int_{0}^{x} b(\xi+\Delta) \psi(\xi+\Delta) d \xi .
$$

From $b(1)>0$ it follows that there is a $\delta \in(0, \Delta)$ and an $\eta>0$ such that $b(x)>\eta$, $x \in(1-\delta, 1]$. 
Now let $p=\nu+\lambda \in(0,1), \varepsilon=\sqrt[p]{\frac{1}{2}} \cdot \delta$ and let $\psi_{p} \in L_{1}^{+}[0,1]$ be given by

$$
\begin{aligned}
& \psi_{p}(x)=(1-x)^{-1+p}, \quad x \in(1-\delta, 1-\varepsilon), \\
& \psi_{p}(x)=0, \quad \text { elsewhere. }
\end{aligned}
$$

From (6.13) it follows that

$$
\left(T_{\lambda} \psi_{p}\right)(x) \geqq C(1-x)^{-1+p} \int_{1-\delta}^{1-\varepsilon} \eta \cdot(1-x)^{-1+p} d x=\frac{C \eta}{2 p} \delta^{p} \psi_{p}(x),
$$
and Lemma 6.4 yields that $r\left(T_{-v+p}\right) \geqq \frac{C \eta}{2 p} \cdot \delta^{p}$, hence $\lim _{\lambda \downarrow-v} r\left(T_{\lambda}\right)=\infty$, so we have
established (6.12) in this case.

(ii) $b(1)=0 . \quad$ Clearly $T_{\lambda} \psi \geqq S_{\lambda} \psi, \quad \psi \in L_{+}^{1}[0,1], \quad v+\lambda>0, \quad$ where $\left(S_{\lambda} \psi\right)(x)$ $=\frac{E_{\lambda}(x)}{g(x)} \int_{0}^{1} h(\xi) \psi(\xi) d \xi$. Therefore $r\left(T_{\lambda}\right) \geqq r\left(S_{\lambda}\right)$. Clearly $1 \in \sigma\left(S_{\lambda}\right)$ for some $\lambda$ with

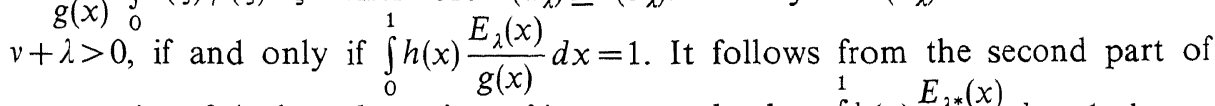
assumption 3.4 that there is a $\lambda^{*}>-v$ such that $\int_{0}^{1} h(x) \frac{E_{\lambda^{*}}(x)}{g(x)} d x=1$, hence $r\left(T_{\lambda^{*}}\right) \geqq r\left(S_{\lambda^{*}}\right) \geqq 1$, and this implies (6.12). Now we have proved

Theorem 6.5. $\omega_{\text {ess }}(T(t))<\omega_{0}(T(t))$.

Remark 6.6. If $b \equiv 0$ and the second part of assumption 3.4 is not fulfilled, then $\omega_{0}(T(t))=\omega_{0}\left(T_{0}(t)\right)=-v$. This can be proved in the following way: suppose $\omega_{0}(T(t))>\omega_{0}\left(T_{0}(t)\right)$. Then there is a $\mu \in \mathbb{C},|\mu|>e^{-v t}$ such that $\mu \in \sigma(T(t))$. Since

$$
\sigma(A) \cap\{\lambda \mid v+\operatorname{Re} \lambda>0\}=\varnothing
$$

and the point spectrum and residual spectrum of the generator $A$ and the semigroup $T(t)$ are faithfull (Pazy (1983)) we may conclude that $\mu$ must be contained in the continuous spectrum of $T(t)$. However this is contradicted by the observation that

$$
\mu I-T(t)=\mu I-T_{0}(t)-U(t)=\left(\mu I-T_{0}(t)\right)\left(I-\left(\mu I-T_{0}(t)\right)^{-1} U(t)\right),
$$

and the compactness of $U(t)$. Hence $\omega_{0}(T(t))=\omega_{0}\left(T_{0}(t)\right)=-v$. In this case we may conclude from (2.6) that for all $\varepsilon>0$ there is a $M(\varepsilon)>0$ such that

$$
\|T(t) \phi\| \leqq M(\varepsilon) e^{-(v-\varepsilon) t}\|\phi\| .
$$

\section{Final Remarks}

In their papers on linear transport theory, Greiner (1984b) and Voigt (1984) also exploit positivity properties of semigroups to determine their asymptotic behaviour, and it is worth mentioning that also in these papers (and other literature on linear transport theory) the inequality $\omega_{\text {ess }}(T(t))<\omega_{0}(T(t))$ plays an important role.

The equations in example 3.8 as well as in example 3.9 induce a semigroup 
$T(t)$ which obeys $\|T(t)\|=1, t \geqq 0$, which says that there is conservation of number. (This can easily be obtained by integrating the equations over all values of $s$.) This yields that

$$
\omega_{\mathrm{ess}}(T(t))<0=\omega_{0}(T(t)) .
$$

One can think of other situations where a similar conservation principle provides an easy proof of the inequality (6.1), and in such cases, positive semigroup theory is extra powerful.

If generations go extinct after finite time (for instance if $g(1)>0$; see Remark 3.6b) then $T_{0}(t)=0, t>\tau(1)$ and hence $T(t)$ is compact, $t>\tau(1)$ implying that $\omega_{\text {ess }}(T(t))=-\infty$, and also in this situation inequality (6.1) is a trivial one. Unfortunately it is now also much more involved to prove irreducibility of the semigroup $T(t)$.

If we drop the assumption that $b$ and $\sigma$ are $C^{1}$ but instead impose the weaker condition

$$
\int_{0}^{1} \frac{|b(x)-\sigma(x)-v|}{g(x)} d x<\infty
$$

then all calculations remain valid.

We expect that the assumption

$$
g(x+\Delta)<g(x), \quad 0<x<1-\Delta,
$$

can be omitted, perhaps at the cost of a strengthening of the second part of assumption 3.4. We refer to Sect. 8 of Diekmann, Heijmans and Thieme (1984), where for a related problem it is shown how a weakening of such an assumption induces (extra) essential spectrum.

If we allow the jump parameter $\Delta$ to take all values between $\Delta_{1}$ and $\Delta_{2}$ where $0<\Delta_{1}<\Delta_{2}<1$, and the probability of making a jump $\Delta$ is determined by the smooth function $p(\Delta), \int_{\Delta_{1}}^{\Delta_{2}} p(\Delta) d \Delta=1$, then (3.1 a) has to be replaced by

$$
\frac{\partial n}{\partial t}(t, x)+\frac{\partial}{\partial x}(g(x) n(t, x))=(\sigma(x)-b(x)) n(t, x)+\int_{\Delta_{1}}^{\Delta_{2}} p(\Delta) b(x+\Delta) n(t, x+\Delta) d \Delta .
$$

In this case the bounded perturbation $C$ (see Sect. 4) is given by

$$
(C \psi)(x)=\int_{\Delta_{1}}^{\Delta_{2}} p(\Delta) b(x+\Delta) \psi(x+\Delta) d \Delta,
$$

and this defines a compact operator. In this case compactness of $U(t)$ follows immediately, and does not require the assumption $g(x+\Delta)<g(x)$. We refer to Heijmans (1984a) for a related problem.

Finally we think it is important to notice that we can avoid the use of Theorem 2.4 and prove Theorem 4.2 exploiting the fact that the semigroup obeys a stronger positivity-condition than irreducibility (cf. Theorem 5.1), namely: for all $\phi \in L_{+}^{1}[0,1], \phi \neq 0$ and $F \in L_{+}^{\infty}[0,1], F \neq 0$ there is a $t=t(\phi, F) \geqq 0$ such that $\langle F, T(t) \phi\rangle>0$ for all $t \geqq t(\phi, F)$. We shall call such a semigroup 
nonsupporting after a concept of Sawashima (1964). Using Sawashima's result on nonsupporting operators we can prove Theorem 2.5, where 'irreducible' is replaced by 'nonsupporting', directly. We refer to Theorem 1.3 of Nussbaum (1984) for a related result.

\section{References}

Birkhoff, G.: Positivity and criticality. Proc. Symp. Appl. Math. 10, 116-126 (1959). Providence: Amer. Math. Soc.

Browder, F.E.: On the spectral theory of elliptic differential operators. Math. Ann. 142, 22-130 (1961)

Diekmann, O., Heijmans, H.J.A.M., Thieme, H.R.: On the stability of the cell size distribution. J. Math. Biol. 19, 227-248 (1984)

Diekmann, O., Heijmans, H.J.A.M., Thieme, H.R.: On the stability of the cell size distribution. II. Time periodic developmental rates. Comp. \& Maths. with Appls. (to appear)

Greiner, G.: Zur Perron-Frobenius-Theorie stark stetiger Halbgruppen. Math. Z. 177, 401-423 (1981)

Greiner, G.: A typical Perron-Frobenius theorem with applications to an age-dependent equation. In: F. Kappel and W. Schappacher (eds.), Infinite Dimensional Systems, Lect. Notes in Math. 1076, 86-100 (1984a)

Greiner, G.: Spectral properties and asymptotic behaviour of the linear transport equation. Math. Z. 185, 167-177 (1984b)

Gripenberg, G.: A stationary distribution for the growth of a population subject to random catastrophes. J. Math. Biol. 17, 371-379 (1983)

Heijmans, H.J.A.M.: An eigenvalue problem related to cell growth. J. Math. Anal. Appl. 111, 253-280 (1985)

Heijmans, H.J.A.M.: On the stable size distribution of a population reproducing by fission into two unequal parts. Math. Biosc. 72, 19-50 (1984a)

Heijmans, H.J.A.M.: Holling's "hungry mantid" model for the invertebrate functional response considered as a Markov process. Part III: The stable satiation distribution. J. Math. Biol. 21, 115-143 (1984b)

Krein, M.G., Rutman, M.A.: Linear operators leaving invariant a cone in a Banach space. Uspehi Mat. Nauk 3: No. 1 (23): 3-95 (1948) [Russian]; English transl.: Am. Math. Soc. Translations (1) 10, 199-325 (1950)

Metz, J.A.J., Batenburg, van F.H.D.: Holling's "hungry mantid" model for the invertebrate functional response considered as a Markov process. Part I: The full model and some of its limits. Part II: Negligable handling time. J. Math. Biol. 22, 209-257 (1985)

Metz, J.A.J., Diekmann, O.: The Dynamics of Physiologically Structured Populations. (in prep.)

Nussbaum, R.D.: The radius of the essential spectrum. Duke Math. J. 38, 473-488 (1970)

Nussbaum, R.D.: Positive operators and elliptic eigenvalue problems. Math. Z. 186, 247-264 (1984)

Pazy, A.: Semigroups of Linear Operators and Applications to Partial Differential Equations. Berlin Heidelberg New York: Springer 1983

Prüss, J.: Equilibrium solutions of age-specific population dynamics of several species. J. Math. Biol. 11, 65-84 (1981)

Sawashima, I.: On spectral properties of some positive operators. Nat. Sci. Dep. Ochanomizu Univ. 15, 53-64 (1964)

Schaefer, H.H.: Banach Lattices and Positive Operators. Berlin Heidelberg New York: Springer 1974

Voigt, J.: Positivity in time dependent linear transport theory. Acta Appl. Math. 2, 311-331 (1984)

Webb, G.F.: Theory of Nonlinear Age-dependent Population Dynamics. New York: Marcel Dekker 1985 